\title{
Identification of System with Bouc-Wen Hysteresis
}

\author{
Nikolay Karabutov ${ }^{1, *}$ \\ ${ }^{1}$ Mirea - Russian Technological University, Department of Problems Control, RU-119454 Moscow, Russia
}

\begin{abstract}
A method of adaptive identification of parameters for a system with Bouc-Wen hysteresis has been developed. The method is based on the use of adaptive observers and resolves the problem of stability of the identification system. Adaptive algorithms of identification were obtained using the Lyapunov second method. The stability proof of the adaptive system is based on the application of Lyapunov vector functions. Adaptive algorithms for adjusting model parameters were developed obtained and finiteness of trajectories in the adaptive system was pointed out.
\end{abstract}

\section{Introduction}

The Bouc-Wen (BWM) model is widely used to describe a hysteresis [1-11]. System with BWM has the form

$$
\begin{gathered}
m \ddot{x}+c \dot{x}+F(x, z, t)=f(t), \\
F(x, z, t)=\alpha k x(t)+(1-\alpha) k d z(t), \\
\dot{z}=d^{-1}\left(a \dot{x}-\beta|\dot{x}||z|^{n} \operatorname{sign}(z)-\gamma \dot{x}|z|^{n}\right),
\end{gathered}
$$

where $m>0$ is mass, $c>0$ is damping, $F(x, z, t)$ is the recovering force, $d>0, n>0, k>0, \alpha \in(0,1), f(t)$ is exciting force, $a, \beta, \gamma$ are some numbers.

Equation (3) is the BWM. Many modifications of BWM [3] have been proposed. Each proposed model considers features of the considered object. The degree of success of the BWM application strongly depends on the identification of its parameters. The solution of the nonlinear equation (3) is the main problem of the BWM identification. A three-level algorithm [4], based on regression analysis, least squares or Gauss-Newton methods, and the extended Kalman filter, is applied to Bouc-Wen model identification. The relevant approach has been applied in [5, 6]. Adaptive algorithms were proposed in $[8,9]$ for the BWM parameters estimation with the data forgetting [7]. Paper [10] presents an adaptive on-line identification methodology with a variable trace method to adjust the adaptation gain matrix.

Examples [11] are known when BWM parameters estimations do not agree with results obtained for other inputs. Such examples confirm the identification ambiguity which causes instability of the model and emphasize that the Bouc-Wen model should be stable in order to provide an adequate description of a physical process [12].
The analysis of literature shows that accounting for features of the object being examined is critically important for the development of algorithms and procedures for the identification of Bouc-Wen model parameters and that. the main difficulties in the estimation of the BWM parameters are related to the model stability and choice of the input. As a rule, the range, in which BWM parameters are varying, should be specified and all the derivatives of the object are should be measured. However, this is not always happenning that makes the application of the proposed algorithms ineffecient.

In this work, the adaptive identification method based on adaptive observer application [13] is used for the solution of the model (3) stability problem. The systemspecified by the equations (1)-(3) is considered. It is assummedthat the input $f(t)$ and the output $x(t)$ are measured.

\section{Problem statement}

Let us consider the system $S_{B W}$ (1)-(3). Let $y$ be the output of the system. The set of the experimental data has the form $\mathrm{I}_{o}=\{f(t), y(t), t \in J\}$, where $J \subset R$ is the given time slice.

Now we designate the parameters vector of the system as $A=[m, c, a, k, \alpha, \beta, \gamma, n]^{T}$.

Problem: to design the adaptive observer for vector estimation $A$ of the system $S_{B W}$ that satisfy the condition

$$
\lim _{t \rightarrow \infty}|\hat{y}(t)-y(t)| \leq \pi_{y},
$$

where $\hat{y} \in R$ is the output of the adaptive observer, $\pi_{y} \geq 0$.

e-mail: kn22@yandex.ru 


\section{About identifiability of $S_{B W}$-system}

The identification effectiveness of the system $S_{B W}$ depends on the features of the input $f(t)$. The requirements to $f(t)$ in identification problems are known. The force $f(t)$ satisfies the condition of constant excitation (CE). This condition is necessary, but not sufficient [14]. The input having the CE property cannot ensure the identifiability of the hysteresis structure. The structural identifiability of the hysteresis is guaranteed with the input $f(t)$ having the Sstabilisation property of the system $S_{B W}$ [14]. Conditions of this property verification are given in [14].

\section{Adaptive system identification}

Let us consider the case $d=1, a=1$. Substitute $F(x, z, t)$ in (1), and divide it by $s+\mu$, where $\mu>0$ and does not coincide with roots of the polynomial $s^{2}+a_{1} s+a_{2}, s=d / d t$. Then transform (1) to the form

$$
\begin{aligned}
\dot{x} & =a_{1} x+a_{2} p_{x}+a_{3} p_{z}+b p_{f}, \\
\dot{p}_{x} & =-\mu p_{x}+x, \dot{p}_{f}=-\mu p_{f}+f, \\
\dot{p}_{z} & =-\mu p_{z}+z,
\end{aligned}
$$

where $\quad a_{1}=-(c-\mu m) / m, \quad a_{2}=-(\alpha k-\mu(c-\mu m) / m$, $a_{3}=-(1-\alpha) k / m$.

Equations (5) and (6) contain only measurable variables, except for $Z$. This complicates the identification process of the system $S_{B W}$ parameters. Now we apply the model

$$
\dot{\hat{x}}=-k_{x}(\hat{x}-x)+\hat{a}_{1} x+\hat{a}_{2} p_{x}+\hat{a}_{3} p_{z}+\hat{b} p_{f}
$$

to the parameters estimation of (5), where $k_{x}>0$ is the specified number; $\hat{a}_{i}(t), i=1,2,3$, and $\hat{b}(t)$ are adjusted parameters.

Let $e=\hat{x}-x$. Obtain the equation for the identification error from (5), (7)

$$
\dot{e}=-k_{x} e+\Delta a_{1} x+\Delta a_{2} p_{x}+\Delta a_{3} p_{z}+\Delta b p_{f},
$$

where $\Delta b=\hat{b}(t)-b, \Delta a_{1}=\hat{a}_{1}(t)-a_{1}, \Delta a_{2}=\hat{a}_{2}(t)-a_{2}$, $\Delta a_{3}=\hat{a}_{3}(t)-a_{3}$.

The (8) is not solvable as the variable $z$ in (6). is unknown Now we shall obtain the current estimation for $z(t)$. Consider model

$$
\dot{\hat{x}}_{\bar{z}}=-k_{x}\left(\hat{x}_{\bar{z}}-x\right)+\hat{a}_{1} x+\hat{a}_{2} p_{x}+\hat{b} p_{f} \text {. }
$$

After that we etermine by the misalignment $\varepsilon_{z}=x-\hat{x}_{\bar{z}}$ and use it for the variable $z$ estimation.
Let $\mathrm{r} \varepsilon_{z}$ be the current estimation $\boldsymbol{Z}$. After applying the model to the estimation of $Z$, we get

$\dot{\hat{z}}=-k_{z}\left(\hat{z}-\varepsilon_{z}\right)+\tilde{\dot{X}}-\hat{\beta}|\tilde{\dot{x}}||\hat{z}|^{n} \operatorname{sign}(\hat{z})-\hat{\gamma} \tilde{\dot{x}}|\hat{z}|^{n}$,

where $\tilde{\dot{x}}=(x(t+\tau)-x(t)) / \tau, \quad k_{z}>0 \quad$ is the given number; $\hat{\beta}, \hat{\gamma}$ are the hysteresis (3) parameters estimations;, $\tau$ is the integration step.

Let us introduce the misalignment $\varepsilon=\hat{z}-\varepsilon_{z}$ and obtain the equation for $\varepsilon$

$$
\begin{gathered}
\dot{\varepsilon}=-k_{z} \varepsilon+\Delta \dot{x}+\Delta \beta|\tilde{\dot{x}}||\hat{z}|^{n} \operatorname{sign}(\hat{z})+\beta \eta_{\beta}+ \\
\Delta \gamma \tilde{\dot{x}}|\hat{z}|^{n}+\gamma \eta_{\gamma}
\end{gathered}
$$

$\eta_{\beta}=|\dot{x}||z|^{n} \operatorname{sign}(z)-|\tilde{\dot{x}}||\hat{z}|^{n} \operatorname{sign}(\hat{z}), \quad \eta_{\gamma}=\dot{x}|z|^{n}-\tilde{\dot{x}}|\hat{z}|^{n}$,

where $\Delta \dot{x}=\tilde{\dot{x}}-\dot{x}, \Delta \beta=\beta-\hat{\beta}, \Delta \gamma=\gamma-\hat{\gamma}$.

Let present (7) as

$$
\dot{\hat{x}}=-k_{x}(\hat{x}-x)+\hat{a}_{1} x+\hat{a}_{2} p_{x}+\hat{a}_{3} p_{\hat{z}}+\hat{b} p_{f},
$$

where

$$
\dot{p}_{\hat{z}}=-\mu p_{\hat{\imath}}+\hat{z} \text {. }
$$

Then, (8) can be rewriten as

$$
\dot{e}=-k_{x} e+\Delta a_{1} x+\Delta a_{2} p_{x}+\Delta a_{3} p_{\hat{z}}+\Delta b p_{f},
$$

and adaptive algorithms is described

$$
\begin{aligned}
& \Delta \dot{a}_{1}=-\gamma_{1} e x, \Delta \dot{a}_{2}=-\gamma_{2} e_{x}, \\
& \Delta \dot{a}_{3}=-\gamma_{3} e p_{\hat{z}}, \Delta b=-\gamma_{b} e p_{f},
\end{aligned}
$$

where $\gamma_{i}>0, i=1,2,3 ; \gamma_{b}>0$.

Tuning algorithms for $\Delta \beta$ and $\Delta \gamma$ into (10) have the following form

$$
\begin{gathered}
\Delta \dot{\beta}=-\chi_{\beta} \varepsilon|\tilde{\dot{x}}||\hat{z}|^{n} \operatorname{sign}(\hat{z}), \\
\Delta \dot{\gamma}=-\chi_{\gamma} \varepsilon \tilde{\dot{x}}|\hat{z}|^{n},
\end{gathered}
$$

where $\chi_{\beta}>0, \chi_{\gamma}>0$ are parameters ensuring a convergence of algorithms.

Several algorithms are applicable for the indicator $n$ estimation in (10). Their effectiveness depends on several factors. The simple algorithm has the form

$$
\dot{\hat{n}}=\left\{\begin{aligned}
&-\gamma_{n} \varepsilon \hat{\beta}|\hat{z}|^{\hat{n}-1} \hat{z} \tilde{\dot{X}}, \text { if }\left|\frac{\varepsilon}{\varepsilon_{z}}\right| \in\left[v_{0}, v_{1}\right], \\
& 0, \text { if }\left|\frac{\varepsilon}{\varepsilon_{z}}\right| \notin\left[v_{0}, v_{1}\right],
\end{aligned}\right.
$$

where $v_{0}, v_{1}$ are given positive numbers, $\gamma_{n}>0$. 
Remark. Stability of the identification procedure is the main problem the solution of the system with BWM. We proposed the method based on the application of adaptive observers. Another solution to the stability problem is to change the structure of the equation (3). We proposed the equation

$$
\dot{z}=d^{-1}\left(-\rho z|\dot{x}|^{\omega}+a \dot{x}-\beta|\dot{x}||z|^{n} \operatorname{sign}(z)-\gamma \dot{x}|z|^{n}\right)
$$

where $\rho>0, \omega>0$. to describe hysteresis.

\section{Properties of adaptive system}

Let consider the subsystem $A S_{X}$ described by and(15). Let $\Delta K(t) \stackrel{\Delta}{=}\left[\Delta a_{1}(t), \Delta a_{2}(t), \Delta a_{3}(t), \Delta b(t)\right]^{T}$,

$$
\begin{gathered}
V_{K}(t) \stackrel{\Delta}{=} 0.5 \Delta K^{T}(t) \Gamma^{-1} \Delta K(t), \\
V(t)=V_{e}(t)+V_{K}(t)
\end{gathered}
$$

where $\Gamma=\operatorname{diag}\left(\gamma_{1}, \gamma_{2}, \gamma_{3}, \gamma_{b}\right)$.

Assumption 1. The input $f(t)$ is constantly exciting and limited.

Theorem 1. Let 1) functions (18), $V_{K}(t)$ are positive definite and satisfy the condition $\inf _{|e| \rightarrow \infty} V_{e}(e) \rightarrow \infty$, $\left.\inf _{\|\Delta K\| \rightarrow \infty} V_{K}(\Delta K) \rightarrow \infty ; 2\right)$ assumption 1 for the system (1)(3) is satisfied. Then all trajectories of the system $A S_{X}$ are limited belong area $\mathrm{G}_{t}=\left\{(e, \Delta K): V(t) \leq V\left(t_{0}\right)\right\}$ and the estimation

$$
\int_{t_{0}}^{t} 2 k_{x} V_{e}(\tau) d \tau \leq V\left(t_{0}\right)-V(t)
$$

is fair.

The theorem 1 shows the limitation of adaptive system trajectories. The asymptotical stability ensuring the system demands to impose additional conditions.

$$
\text { Let } P(t) \triangleq\left[x(t) p_{x}(t) p_{\hat{z}}(t) p_{f}(t)\right]^{T} \text {. }
$$

Definition 1. The vector $P$ is constantly excited with a level $v$ or have property $P E_{v}$ if

$$
P E_{v}: P(t) P^{T}(t) \geq v I_{4}
$$

fairly for $\exists v>0$ and $\forall t \geq t_{0}$ on some interval $T>0$, where $I_{4} \in R^{4}$ is the unity matrix.

If the vector $P(t)$ has property $P E_{v}$ then we will write $P(t) \in P E_{v}$.

The system $S_{B W}$ is stable, and the input $f(t)$ is restricted. Therefore, present the property $\mathcal{P} E_{v}$ for the matrix $B_{P}(t)=P(t) P^{T}(t)$ as

$$
P E_{v, \bar{v}}: v I_{4} \leq B_{P}(t) \leq \bar{v} I_{4} \quad \forall t \geq t_{0},
$$

where $\bar{v}>0$ is some number.

Let us the estimation to $V_{K}(t)$ be fair

$$
0.5 \beta_{l}^{-1}(\Gamma)\|\Delta K(t)\|^{2} \leq V_{K}(t) \leq 0.5 \beta_{1}^{-1}(\Gamma)\|\Delta K(t)\|^{2},(21
$$

where $\beta_{1}(\Gamma), \quad \beta_{l}(\Gamma)$ are minimum and maximum eigenvalues of the matrix $\Gamma$.

Now we apply inequalities (20), (21) and obtain estimations for $\dot{V}_{e}, \dot{V}_{K}$

$$
\begin{gathered}
\dot{V}_{e} \leq-k_{x} V_{e}+\frac{\bar{v} \beta_{l}(\Gamma)}{k_{x}} V_{K}, \\
\dot{V}_{K} \leq-\frac{3}{4} \vartheta v \beta_{1}(\Gamma) V_{K}+\frac{8}{3} \vartheta V_{e},
\end{gathered}
$$

Estimates (22), (23) are obtained applying the approach [15].

Theorem 2. Let conditions be satisfied 1) positive definite Lyapunov functions $V_{e}(t)$ and $V_{K}(t)$ allow the indefinitely small highest limit at $|e(t)| \rightarrow 0$, $\|\Delta K(t)\| \rightarrow 0$; 2) $P(t) \in P E_{v, \bar{v}}$; 3) equality

$$
e \Delta K^{T} P=\vartheta\left(\Delta K^{T} B \Delta K+e^{2}\right)
$$

is fair in the area $O_{v}(O)$ with $0<\vartheta$, where $O=\left\{0,0^{3 m}\right\} \subset R \times R^{3 m} \times J_{0, \infty}, \quad O_{v} \quad$ is some neighbourhood of the point $O$; 4) the function $V_{K}(t)$ satisfies (21); 5) $\dot{V}_{\varepsilon}, \dot{V}_{K}$ satisfy the system of inequalities

$$
\left[\begin{array}{c}
\dot{V}_{e} \\
\dot{V}_{K}
\end{array}\right] \leq \underbrace{\left[\begin{array}{cc}
-k_{x} & \frac{\bar{v} \beta_{l}(\Gamma)}{k_{x}} \\
\frac{8}{3} \vartheta & -\frac{3 v \vartheta \beta_{1}(\Gamma)}{4}
\end{array}\right]}_{A_{V}}\left[\begin{array}{c}
V_{e} \\
V_{K}
\end{array}\right] ;
$$

$6)$ the upper solution for $V_{e, K}(t)=\left[V_{e}(t) V_{K}(t)\right]^{T}$ satisfies to the comparison equation $\dot{S}=A_{v} S$ if

$$
V_{\rho}(t) \leq s_{\rho}(t) \quad \forall\left(t \geq t_{0}\right) \&\left(V_{\rho}\left(t_{0}\right) \leq s_{\rho}\left(t_{0}\right)\right),
$$

where $\rho=e, K, S=\left[s_{e} s_{K}\right]^{T}, A_{v} \in R^{2 \times 2}$ is $M$-matrix. Then the system $A S_{X}$ is exponentially stable with the estimation

$$
V_{e, K}(t) \leq e^{A_{V}\left(t-t_{0}\right)} S\left(t_{0}\right),
$$

if

$$
k_{x} \geq \frac{4}{3} \sqrt{\frac{2 \bar{v} \beta_{l}(\Gamma)}{v \beta_{1}(\Gamma)}} .
$$


Theorem 2 shows that the adaptive system $A S_{X}$ gives the true estimates for parameters of the system (1). This is fair at the fulfilment of conditions (24). We assume that the variable $p_{\hat{z}}$ restricted.

The boundedness of the variable $\hat{X}_{\bar{z}}$ follows from the boundedness of the system $A S_{X}$ trajectories.

Let us consider subsystem $A S_{Z}$ described by equations (12), (16) and

introduce Lyapunov functions

$$
\begin{aligned}
& V_{\varepsilon \beta \gamma}(t)=V_{\varepsilon}(t)+V_{\beta, \gamma}(t), \\
& V_{\beta, \gamma}(t)=0.5 \chi_{\beta}^{-1}(\Delta \beta(t))^{2}+0.5 \chi_{\gamma}^{-1}(\Delta \gamma(t))^{2} .
\end{aligned}
$$

\section{Theorem 3. Let}

(1) functions $V_{\varepsilon}(t)=0.5 \varepsilon^{2}(t), \quad V_{\beta, \gamma}(t)$ are positive definite and satisfy conditions

$$
\inf _{|\varepsilon| \rightarrow \infty} V_{\varepsilon}(\varepsilon) \rightarrow \infty, \inf _{\|[\Delta \beta, \Delta \gamma]\| \rightarrow \infty} V_{\beta, \gamma}(\Delta \beta, \Delta \gamma) \rightarrow \infty ;
$$

(2) the function $V_{\varepsilon \beta \gamma}(t)$ has the form (25); 3) the function

$$
\tilde{g}_{1}(t)=\sup _{\varepsilon \in \Omega} \frac{|\varepsilon|^{n+1}(t)}{V_{\varepsilon}(t, \varepsilon)}, g_{1}=\sup _{\varepsilon \in \Omega} \tilde{g}_{1}(t),
$$

exists, where $\Omega$ is the definition range of the subsystem $A S_{Z}$; (4) $|\Delta \dot{x}| \leq \delta_{\Delta}, \delta_{\Delta} \geq 0$; 5) $|\dot{x}| \leq v, v>0$; 6) the assumption 1 holds for the system (1)-(3). Then all trajectories of the system $A S_{Z}$ are bounded, belonging to the area $\mathrm{G}_{\varepsilon}=\left\{(\varepsilon, \Delta \beta, \Delta \gamma): V_{\varepsilon \beta \gamma}(t) \leq V_{\varepsilon \beta \gamma}\left(t_{0}\right)\right\}$, and the estimation

$$
\begin{aligned}
& \int_{t_{0}}^{t}\left(k_{z}-v(\beta+\gamma) g_{1}\right) V_{\varepsilon}(\tau) d \tau+ \\
& \frac{1}{2\left(k_{z}-v(\beta+\gamma) g_{1}\right)\left(t-t_{0}\right)}\left(\delta_{\Delta}\right)^{2} \leq \\
& V_{\varepsilon \beta \gamma}\left(t_{0}\right)-V_{\varepsilon \beta \gamma}(t)
\end{aligned}
$$

is fair if

$$
k_{z}>v(\beta+\gamma) g_{1} \text {. }
$$

Hence, the boundedness of trajectories in the adaptive system is proved. The analysis showed that the subsystem $A S_{X}$ is asymptotically stable. The prove of trajectories boundedness for the subsystem $A S_{Z}$ is a more complex problem in parametrical and output spaces. This problem is solvable if the condition (28) is satisfied. The estimation (27) shows that the quality of processes in the $A S_{Z}$-system depends on the output derivative of the $S_{B W}$-system. The following result given more exact estimations for processes in the $A S_{Z}$-system.
Theorem 4. Let (1) positive definite Lyapunov functions $V_{\beta, \gamma}(t)$ and $V_{\varepsilon}(t)$ allow the indefinitely small higher

limit $\quad\|[\Delta \beta(t), \Delta \gamma(t)]\| \rightarrow 0 \quad$ to $\quad|\varepsilon(t)| \rightarrow 0$;

$P(t) \in P E_{v, \bar{v}}$; (3) such $c_{1}>0, c_{2}>0$ exist that conditions

$$
\begin{gathered}
\varepsilon \Delta \gamma \tilde{\dot{x}}|\hat{z}|^{n}=c_{2}\left[(\Delta \gamma)^{2}\left(\tilde{\dot{x}}|\hat{z}|^{n}\right)^{2}+\varepsilon^{2}\right], \\
\varepsilon \Delta \beta|\tilde{\dot{x}}||\hat{z}|^{n} \operatorname{sign}(\hat{z})=c_{1}\left[(\Delta \beta)^{2}\left(|\tilde{\dot{x}}||\hat{z}|^{n}\right)^{2}+\varepsilon^{2}\right]
\end{gathered}
$$

are satisfied in the area $O_{v}(O)$, where $O=\left\{0,0^{2}\right\} \subset R \times R^{2} \times J_{0, \infty}, O_{v}$ is some neighbourhood of the point $O$; (4) inequality $\left(\varepsilon-\varepsilon_{z}\right)^{2 n} \geq c_{z}$ holds for almost all $t$ where $c_{z} \geq 0$; (5) such $\pi_{x} \geq 0$ and $\omega>0$ exist that $(\tilde{\dot{x}})^{2} \geq \pi_{x}$ и $\left|\varepsilon-\varepsilon_{z}\right| \leq \omega|\varepsilon|$; (6) the function

$$
g_{2}(t)=\sup _{\varepsilon \in \Omega} \frac{|\varepsilon|^{2(n+1)}(t)}{V_{\varepsilon}(t, \varepsilon)}, g_{2}=\sup _{\varepsilon \in \Omega} \tilde{g}_{2}(t)
$$

exists, where $\Omega$ the subsystem $A S_{Z}$ definition domain; (7) $\dot{V}_{\varepsilon}, \dot{V}_{\beta, \gamma}$ satisfy the system of inequalities

$$
\begin{aligned}
{\left[\begin{array}{c}
\dot{V}_{\varepsilon} \\
\dot{V}_{\beta, \gamma}
\end{array}\right] \leq } & \underbrace{\left[\begin{array}{cc}
-\left(k_{z}-2 \tilde{v} g_{1}-\omega v g_{2}\right) & \lambda \chi \omega v \\
c & -\frac{d_{s}}{2}
\end{array}\right]}_{A_{\varepsilon}}\left[\begin{array}{c}
V_{\varepsilon} \\
V_{\beta, \gamma}
\end{array}\right]+ \\
& \underbrace{\left[\begin{array}{c}
\frac{1}{2 k_{z}} \\
0
\end{array}\right]}_{B_{\varepsilon}}\left(\delta_{\Delta}\right)^{2} ;
\end{aligned}
$$

(8) the upper solution for $V_{\varepsilon, \beta, \gamma}=\left[V_{\varepsilon}(t) V_{\beta, \gamma}(t)\right]^{T}$ satisfies to the equation

$$
\dot{\tilde{S}}=A_{\varepsilon} \tilde{S}+B_{\varepsilon}\left(\delta_{\Delta}\right)^{2}
$$

If

$$
V_{\tilde{\rho}}(t) \leq \tilde{s}_{\tilde{\rho}}(t) \quad \forall\left(t \geq t_{0}\right) \&\left(V_{\tilde{\rho}}\left(t_{0}\right) \leq \tilde{s}_{\tilde{\rho}}\left(t_{0}\right)\right)
$$

where $\tilde{\rho}=\varepsilon, \beta, \gamma, \tilde{S}=\left[\tilde{S}_{\varepsilon} \tilde{s}_{\beta, \gamma}\right]^{T}, A_{\varepsilon} \in R^{2 \times 2}$ is $M$ matrix. Then the system $A S_{Z}$ is exponentially dissipative with the estimation

$$
V_{\varepsilon, \beta, \gamma}(t) \leq e^{A_{\varepsilon}\left(t-t_{0}\right)} \tilde{S}\left(t_{0}\right)+\left(\delta_{\Delta}\right)^{2} \int_{t_{0}}^{T} e^{A_{\varepsilon}(t-\tau)} B_{\varepsilon} d \tau,
$$

if $\left(k_{z}-2 \tilde{v} g_{1}-\omega v g_{2}\right) d_{s}>2 c \lambda \chi \omega v, k_{z}>2 \tilde{v} g_{1}-\omega v g_{2}$, $d_{s}>0$, 


$$
\begin{aligned}
& \bar{\chi}=\min \left(\chi_{\beta}, \chi_{\gamma}\right), \bar{c}=\min \left(c_{1}, c_{2}\right), \\
& \chi=\max \left(\chi_{\beta}, \chi_{\gamma}\right), d_{s}=\chi \pi_{x} \bar{c} c_{z} .
\end{aligned}
$$

We have shown that the system $A S_{Z}$ is exponentially dissipative. The area of the dissipativity depends on the informational set $\mathrm{I}_{O}$ of the $S_{B W}$-system. The obtained results justify the application of adaptive observers for the $S_{B W}$-system identification.

\section{Simulation results}

Let us consider the system (5)-(8) with parameters $n=1.5, c=2, m=1, \beta=0.5, \alpha=0.7, k=0.6$. Let $d=a=1$. The exciting force $f(t)=2-2 \sin (0.15 \pi t)$. The system $S_{B W}$ modelled with initial conditions $x(0)=1, \dot{x}(0)=0, z(0)=1$. Form the set $\mathrm{I}_{o}$. The system phase portrait and output of the hysteresis are shown in Fig. 1.

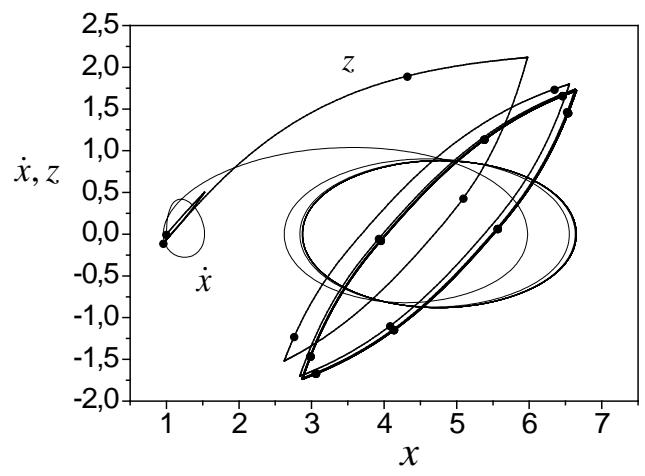

Fig. 1. System phase portrait and hysteresis change.

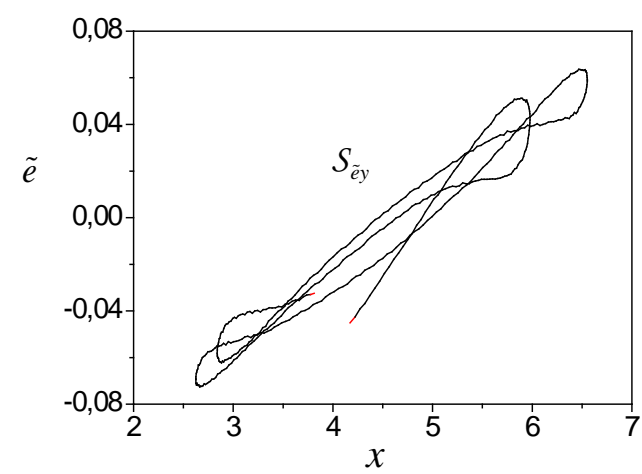

Fig. 2. System phase portrait and hysteresis change.

Let us construct the framework $S_{\tilde{e} y}$ (Fig. 2), using the method [14], and evaluate the structural identifiability of the system $S_{B W}$. The variable $\tilde{e} \in R$ is equal $\tilde{e}=\dot{x}-\hat{\dot{x}}_{h} . \hat{\dot{x}}_{h}$ is the estimation of the steady state (process) in the $S_{B W}$-system for $\forall t \geq 9.85_{\mathrm{s}}$, and $\tilde{e}$ is the hysteresis output estimation. Fig. 1 and 2 show that definition ranges $z$ and $\tilde{e}$ coincides. The analysis of $S_{\tilde{e} y}$ shows that the system $S_{B W}$ is structurally identifiable, and the input $f(t)$ is S-stabilizing.

Let usonsider the identification of the system $S_{B W}$ parameters. After that we determine by the parameter $\mu$ of the system Eq. (13) using the transient process analysis for $\tilde{e}$ and $t<9.85 \mathrm{~s}$. Now we alculate Lyapunov exponents (LE) [16]. The estimation for the maximum LE is -0.9 . Therefore, we set $\mu=0.8$. Initial conditions in (6) are equal to zero.

The results of the work of the adaptive system are presented in Figs. 3-7. Parameters $k_{x}, k_{z}$ are equal to 2.5 and 0.75 . The tuning process of $A S_{X}$-systems (the model (7)) parameters are shown in Fig. 3, Fig. 4 shows the tuning parameters of the model (10).

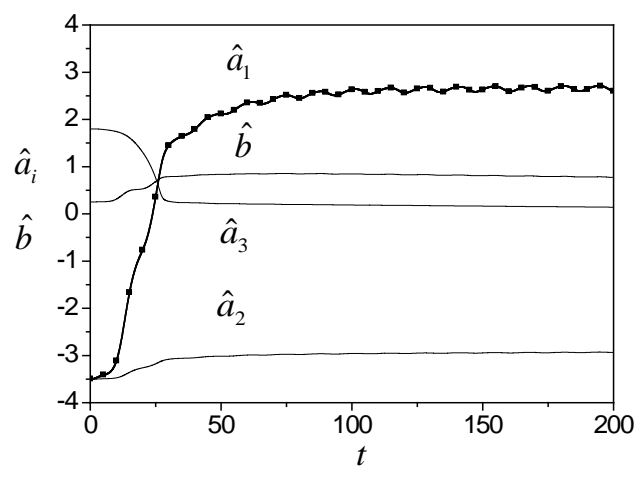

Fig. 3. Tuning of model (7) parameters.

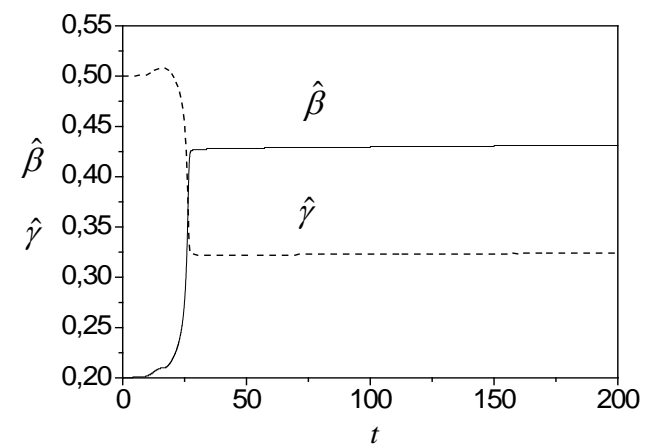

Fig. 4. Tuning of model (10) parameters.

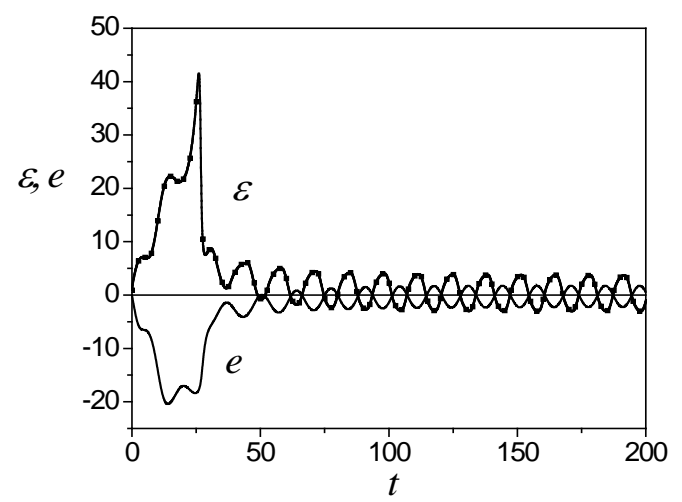

Fig. 5. Outputs modification of systems $A S_{X}, A S_{Z}$. 
The modification of identification errors $e, \varepsilon$ are shown in Fig. 5. We can see that the accuracy of obtained estimations depends on numbers of tuned parameters and the $\dot{x}$ level, and properties $f(t)$. Obtained results confirm statements of theorems 3, 4 . The results of $A S_{Z}$-system work influence the tuning processes in the $A S_{X}$-system. Gain coefficients in (15), (16) and (17) are $\chi_{\beta}=0.0000002, \chi_{\gamma}=0.0000002$, $\gamma_{4}=0.00005 \gamma_{1}=0.0002, \gamma_{2}=0.00001, \gamma_{3}=0.00002$ Fig. 6.

The hysteresis output estimation process is shown in

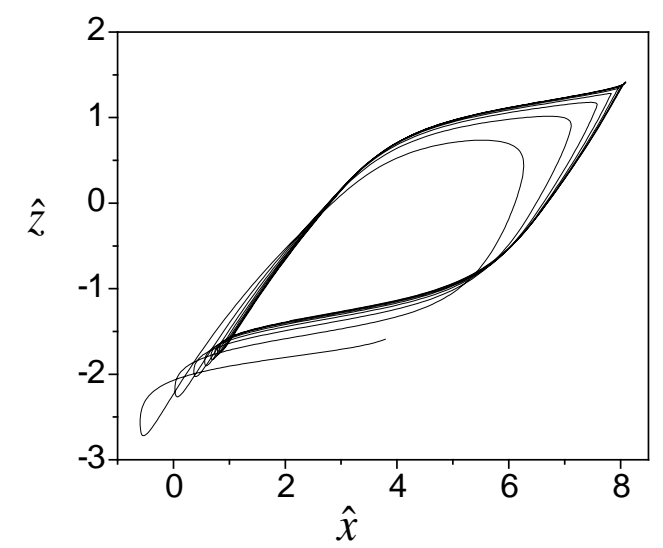

Fig. 6. Hysteresis estimation at adaptation of $A S_{B W}$-system

So, simulation results confirm the exponential dissipativity of the designed system.

\section{Conclusion}

The adaptive parameter identification method for the system with Bouc-Wen hysteresis has been developed. The method is based on the application of adaptive observers and solves the stability problem. Adaptive algorithms of model parameter tuning have been designed, and the boundedness of trajectories in the adaptive system is shown. Estimates of uncertainty, which are used for the tuning of the hysteresis model parameters, have been obtained.

We show that the boundary of the system exponential dissipativity area is determined by the system output derivative level.

\section{References}

1. R. Bouc, Proceedings of the Fourth Conference on Nonlinear Oscillations, (Prague, Czechoslovakia 1967).

2. Y.K. Wen, J. Eng. Mech. Div., 102, 2 (1976).

3. M. Ismail, F. Ikhouane, and J. Rodellar, Archives of Computational Methods in Engineering, 16, (2009).
4. C. H. Loh, and S.T. Chung, Earthquake Engineering \& Structural Dynamics, 22, (1993).

5. S.K. Kunnath, J.B. Mander, L. Fang, Engineering Structures, 19(3) (1997).

6. T.T. Baber, Y.K. Wen, Journal of Engineering Mechanics, 107(6) (1981).

7. P.A. Ioannou, and J. Sun, Robust adaptive control, (New York: Prentice Hall. 1996).

8. A.G. Chassiakos, S.F. Masri, A.W. Smyth, and T.K. Caughy, J. Appl. Mech., 65 (1998).

9. A.W. Smith, S.F. Masri, A.G. Chassiakos, and T.K. Caughey, Journal of Engineering Mechanics, 125(2) (1999).

10. J.W. Lin, R. Betti, A.W.Smyth, and R.W. Longman, Earthquake Engineering and Structural Dynamics, 30 (2001).

11. A.N. Danilin, E.L. Kuznetsova, N.N. Kurdumov, L.N. Rabinsky, and S.S. Tarasov, PNRPU Mechanics Bulletin, 4 (2016).

12. M. Ismail, F. Ikhouane, and J. Rodellar, Archives of Computational Methods in Engineering, 16 (2009).

13. N. Karabutov, In Advances in Engineering Research. Volume 28, (New York: Nova Science Publisher 2019).

14. N. Karabutov, Global Journal of Science Frontier Research: (A) Physics and Space Science, 18, 11 (2018).

15. N. Karabutov, International Journal of Intelligent Systems and Applications, 10(1) (2018).

16. N. Karabutov, International journal of intelligent systems and applications, 7(1) (2015). 